of concurrent viral plaques. Despite rapid cell-to-cell spread of HSV2 , infected cells are eliminated by localised CD8 + T-cells within $24 \mathrm{~h}$ of plaque initiation. Moreover, the extent of secondary plaque formation prior to episode termination is determined by spatial CD8 + T-cell density surrounding the site of infection.

Conclusions Genital HSV-2 utilises three kinetically distinct methods of spread to initiate and sustain prolonged shedding episodes. The extent and severity of secondary plaque formation is determined by spatial immune cell density.

\section{4-S1.05 RNA HELICASES, P72 AND P68 AND HDAC1 INTERACT WITH HIV-1 INTEGRASE AND AFFECT VIRAL REPLICATION}

doi:10.1136/sextrans-2011-050109.143

F Larguet, É Rassart, E Edouard. Université du Québec à Montréal, Montreal, Canada

Background Human immunodeficiency virus type-1 (HIV-1) infection is one of the leading causes of death worldwide. Current antiHIV-1 therapy, referred as highly active antiretroviral therapy (HAART), is based on the use of combination of drugs directed against viral enzymes mainly reverse transcriptase and protease and more recently integrase. Indeed, HAART has dramatically improved the clinical course of the disease. However, the emergence of multidrug resistant virus strains during treatment highlights the urgent need to develop novel antiretroviral drugs against new HIV-1 targets. HIV-1 is able to hijack cellular machinery for its replication through protein-protein interactions between viral and host cell factors and a rising strategy against HIV-1 infection is to inhibit key virus-cell interactions. Integrase that catalyses HIV-1 viral DNA integration into the host cell genome is currently a focus for the development of new drugs. Several cellular partners of integrase have been identified using different methods. Based on a different strategy, our study aimed to identify new integrase cellular partners. Methods We used a biotinylated oligonucleotide derived from the viral U3 LTR end as bait to isolate integrase in a streptavidin beads magnetic separation. Proteins co-purified with integrase were analysed by mass spectrometry.

Results Interestingly, our method allowed the identification of new cellular proteins notably p72 and p68 RNA helicases and histone deacetylase 1 (HDAC1) as integrase partners in addition to proteins already reported in literature. The interaction of p72, p68 and HDAC1 proteins with integrase was confirmed by co-immunoprecipitation. In addition, specific knockdown of p72 and p68 RNA helicases and HDAC1 were shown to affect HIV-1 replication.

Conclusions Our data suggest that cellular proteins, p72 and p68 RNA helicases and HDAC1 facilitate HIV-1 replication through interaction with integrase.

\section{4-S1.06 HPV 16 PREDICTS CLINICAL OUTCOME IN ORAL CANCER PATIENTS TREATED BY RADIOTHERAPY}

doi:10.1136/sextrans-2011-050109.144

R M Apolinario, P C Lara, L A Henriquez, M Lloret, B Pinar. Las Palmas University Hospital-Canarian Institute for Cancer Research (ICIC), Las Palmas GC, Spain

Growing molecular and clinical evidence indicates that human papillomavirus (HPV) is involved in the aetiology of oral squamous cell carcinomas (SCCs). HPV (+) tumours appear to be clinically distinct from $\mathrm{HPV}(-)$ tumours, conferring improved survival outcomes for patients in oropharyngeal cancer but limited knowledge exist on oral cancer. Determination of the HPV status of tumours may assist in patient risk-stratification and ultimately guide optimum treatment. The primary aim of this study was to examine the distribution of HPV in oral SCCs as assessed in vitro amplification assays and correlated with clinical and demographic data. The secondary aim was to correlate the positivity of HPV tumours with clinical outcome in the largest series of oral cancer published with a long follow-up (up to 20 years).

Materials and Methods One hundred thirty-one invasive oral SCCs were tested for HPV using laboratory-developed PCR assays for HPV16. P53 expression, tumour angiogenesis (CD-31 staining) and proliferation (MIB-1) were also assessed by immunohistochemistry in parafine embedded tissue. Patients mean age was 58.09 \pm 10.41 , median 59 (116 men and 15 women). Clinical Stage distribution was: st I:17 cases; II: 56,III:32.IV:26. Most tumours were histological grade I (39) and II (74). Patients with pathological stage I-II were refereed to surgery (65 cases) and patients with Stage III-IVA were referred to surgery and postoperative radiation therapy (66 cases). Mean radiotherapy given doses were $62.13 \pm 7.74$, median $65 \mathrm{~Gy}$ in 1.8-2 Gy fractions. No chemotherapy was used in any case.

Results 41 cases $(31.3 \%)$ were HPV $16(+)$. No relation was found with age, gender, or tumour characteristics. In fact no relation was found to p53 expression, tumour proliferation or angiogenesis. 15year DFS was $62.20 \%$ in $\mathrm{HPV}(+)$ patients was, compared to $37.3 \%$ in the HPV(-) group $(p<0.076)$. In stage III-IV cases (treated by surgery and radiation therapy) this differences reached statistical signification ( 15 y DFS $72.4 \%$ vs $36.0 \%$ p <0.020). Similar results were found for Cause Especific Survival (15 y DFS 68.4\% vs $26.2 \%$ $\mathrm{p}<0.054)$.

Conclusion These data show that the HPV status is a good predictor of DFS and survival in patients treated with radical surgery and adjuvant radiotherapy in oral carcinomas. This prognostic advantage seem to be independent of tumour proliferation, p53 status or angiogenesis. Other molecular processes could be implicated in the different response to radiotherapy.

\section{Basic sciences oral session 2-Immunity and animal models \\ 04-S2.01 THE HOST RESPONSE TO CHLAMYDIAL INFECTION RESULTS IN INCREASED GONOCOCCAL COLONISATION IN A NOVEL COINFECTION MODEL}

doi:10.1136/sextrans-2011-050109.145

${ }^{1} \mathrm{~A}$ Jerse, ${ }^{1} \mathrm{R}$ Vonck, ${ }^{2} \mathrm{~T}$ Darville. ${ }^{1}$ Uniformed Services University, Bethesda, USA ${ }^{2}$ University of Pittsburgh Medical Center, Pittsburgh, USA

Objective Neisseria gonorrhoeae and Chlamydia trachomatis cause similar urogenital diseases and up to $70 \%$ of individuals with gonorrhoea also have chlamydia. Using a newly developed female mouse model of coinfection, we recently reported that higher numbers of $N$ gonorrhoeae were recovered from mice with a preexisting Chlamydia muridarum infection, the mouse strain of Chlamydia, compared to mice infected with $N$ gonorrhoeae alone. Recent studies on the host response to $N$ gonorrhoeae implicate toll-like receptor 4 (TLR4) and IL17 responses as being protective against $N$ gonorrhoeae. Here we tested the hypothesis that the immune response to chlamydial infection makes the genital tract more permissive to $N$ gonorrhoeae.

Methods Using an immune-targeted RT-PCR array we screened for alterations in host gene expression during chlamydial infection of $\mathrm{BALB} / \mathrm{c}$ mice that may account for the observed increase in gonococcal colonisation. Mouse genital cells were collected by vaginal swab and analysed for TLR4 expression by flow cytometry. Coinfection studies were performed in BALB/cJ (TLR4 wild type) and C. C3-TLR4LPS-d/J (TLR4 mutant) mice and the number of viable chlamydiae and gonococci recovered from each group was determined by immunofluorescence using $L 929$ cells and quantitative culture on GC agar, respectively. 